\title{
Gastric mucous neck cell and intestinal goblet cell phenotypes in gastric adenocarcinoma
}

\author{
N R Hughes, P S Bhathal
}

\begin{abstract}
Aim-To investigate the phenotype of cells comprising diffuse and intestinal-type gastric cancers using monoclonal antibodies to two antigens. One antigen (designated D10) is characteristic of gastric mucous neck cells, cardiac glands, pyloric glands, and Brunner's glands. The second antigen (designated $17 \mathrm{NM}$ ) is specific to the mucous vacuole of intestinal goblet cells.
\end{abstract}

Methods-Thirty two gastrectomy specimens with adenocarcinoma were studied. Serial paraffin sections were stained immunohistochemically for D10 and 17NM and histochemically for acid and neutral mucins. The cancers were classified histologically as of either diffuse or intestinal type according to Lauren.

Results-Of 15 diffuse-type gastric carcinomas, 11 showed the majority of cancer cells staining for D10 while four were typical signet ring cell cancers staining predominantly for 17NM; five tumours displayed both phenotypes with the two phenotypes segregated in different areas of the tumours. In contrast, of 16 intestinal-type cancers, six expressed $17 N M$, three D10, five neither antigen, and two expressed both antigens. One indeterminate-type cancer expressed both antigens. The staining of individual cells for D10 and 17NM was mutually exclusive in both diffuse and intestinal types. In contrast to the diffuse cancers, intestinaltype cancers typically expressed either antigen only in occasional small groups of cells and individual cells.

Conclusions-In disease, the gastric stem cell can assume the capacity of the duodenal stem cell for divergent differentiation into either intestinal goblet cells (for example, as in intestinal metaplasia) or Brunner's gland cells (for example, as in pyloric gland/Brunner's gland metaplasia). With neoplastic transformation, this potential for divergent differentiation is maintained and gives rise to diffuse-type cancers that display either the D10 phenotype, the 17NM phenotype, or the clonal expression of both phenotypes. In the more cell cohesive (intestinal-type) tumours, differentiation for antigen expression is poorly developed and more frequently directed towards the intestinal goblet cell phenotype.

(f Clin Pathol 1997;50:741-748)
Keywords: gastric carcinoma; mucous neck cells; intestinal goblet cells

The histological classification of gastric adenocarcinomas into Lauren's diffuse or intestinal type $^{1}$ has some biological significance as the intestinal-type cancer shows variation in geographical incidence and has a somewhat different clinical behaviour to the diffuse type. ${ }^{23}$ Although intestinal metaplasia is common in stomachs bearing intestinal-type cancers, ${ }^{4}$ the cell lineages of the two classes of tumour and the reason for the frequent histological and histochemical heterogeneity seen in individual specimens of gastric carcinoma remain unclear. We report on the use of two phenotypic markers that identify two distinct neoplastic cell populations in gastric carcinoma and throw some light on the histogenesis of these tumours.

\section{Methods}

Tissues were collected over a four year period from gastrectomy specimens (as approved by the Ethics Committee of The Royal Melbourne Hospital) from 22 men (28-80 years old) and 10 women (45-82 years old) admitted to this hospital for surgery for gastric adenocarcinoma. The specimens were consecutive and unselected except that the fresh specimen needed to be available immediately after resection, and the duty pathologist was prepared to release tissue for the research after diagnostic needs had been met. Blocks of tumour and adjacent mucosa and, when possible, long strips of non-involved mucosa were taken from the fresh specimens for this study. In addition, sections of the cancers were taken subsequently from the routinely processed paraffin blocks used in producing the surgical pathology report.

For optimal antigen preservation, the fresh tissue was either fixed for three to four hours in cold fixative containing $2 \%$ paraformaldehyde, $75 \%$ ethanol, and $0.4 \%$ cetylpyridinium chloride (EPF), or in Zamboni's fixative ${ }^{5}$ for one hour followed by a further three hours in EPF. Tissues were dehydrated in isopropanol, cleared in chloroform, and vacuum embedded in four changes of paraffin wax over a period of one hour. The long strips of mucosa were fixed, dissected free from the muscularis propria, fashioned into "Swiss rolls", and processed for sectioning as described above.

Paraffin sections of four signet ring cell cancers of the caecum and sigmoid colon, adenocarcinomas of the ascending colon (one case) 
and sigmoid colon (four cases) obtained from routinely processed tissue were also studied.

Two micron thick serial paraffin sections were stained immunohistochemically for antigens D10, 17NM, and the antigen detected by the monoclonal antibody 7HGM-1D7-B; in addition, the sections were stained histochemically for acid and neutral mucins (AB-dPAS stain).

PRODUCTION OF MONOCLONAL ANTIBODIES

Preparation of hybridoma 5HL-5D11-D10, which recognises antigen $\mathrm{D} 10$, has been described elsewhere. ${ }^{6}$ The hybridoma was grown intraperitoneally in BALB/c mice and the same batch of ascites fluid used for the previous study was used in the present study.

Hybridoma 17NM-20-20 recognises antigen $17 \mathrm{NM}$ and was produced by immunising mice with isolated colonic glands from subjects with colonic carcinoma; the procedures for preparing cell hybrids, screening, and cloning this hybridoma have been described elsewhere. ${ }^{7}$ The same batch of ascites fluid used in the previous study was used here.

A third monclonal antibody (7HGM1D7-B) used in this study was produced by immunising $\mathrm{BALB} / \mathrm{c}$ mice with epithelium isolated from surgically resected human gall bladders. Cell hybrids were prepared from the spleens of these mice and screened against a mosaic of tissues as described for antibody 5HL-5D11-D10. ${ }^{6}$ Hybridoma 7HGM-1D7-B was selected, cloned by limiting dilution, grown intraperitoneally in BALB/c mice, and the batch of the resulting ascites fluid used here.

\section{IMMUNOHISTOCHEMISTRY}

A four stage immunohistochemical staining procedure was used as previously described. ${ }^{6}$ Briefly, sections were incubated sequentially in Tris buffer $(140 \mathrm{mM} \mathrm{NaCl}, 50 \mathrm{mM}$ Tris, $2.7 \mathrm{mM} \mathrm{KCl}, 0.01 \%$ merthiolate, $\mathrm{pH} 7.2$ ) containing $10 \%$ fetal calf serum (FCS), mouse monoclonal antibody, rabbit immunoglobulins to mouse immunoglobulins (Z259; Dako, Botany, Australia), $1.5 \% \mathrm{H}_{2} \mathrm{O}_{2}$ in Tris buffer, swine immunoglobulins to rabbit immunoglobulins (Z196; Dako) and rabbit horseradish peroxidase antiperoxidase (Z113; Dako). The substrate 3-3'-diaminobenzidine (DAB) was used to demonstrate the sites of bound enzyme. All immunological reagents were diluted in Tris buffer containing $10 \% \mathrm{FCS}$ and $0.1 \% \mathrm{NaN}_{3}\left(0.04 \% \mathrm{NaN}_{3}\right.$ was used with the peroxidase antiperoxidase solution) and the sections were washed between changes for 10 minutes in Tris buffer. The three monoclonal antibodies were all used at a dilution of $1 / 400$. A 1/400 dilution of ascites fluid produced by a non-reactive mouse hybridoma or the Tris buffer containing $10 \%$ FCS were used as negative controls.

To obtain maximum staining of antigens in paraffin sections of the routinely fixed and processed tissue, sections were taken down to water, placed in $0.05 \mathrm{M}$ citrate buffer, $\mathrm{pH} 6$, which had been brought to the boil in a microwave oven, again heated to boiling point in the oven over one minute, and allowed to cool for 20-30 minutes before staining. ${ }^{8}$ Following immunohistochemical staining, sections were counter stained for five minutes with $1 \%$ Alcian blue in 3\% acetic acid (pH 2.5) for acid mucins and then with haematoxylin.

Double staining for D10 and proliferating cell nuclear antigen (PCNA) was done by sequentially reacting sections with a $1 / 500$ dilution of antibody to PCNA (Cat. No. ABT 152; American Biotech, Florida, USA), rabbit antibodies to mouse immunoglobulin (Z259; Dako), and gold labelled goat antibodies to rabbit immunoglobulins (Cat. No. RPN 470; Amersham International, Amersham, UK), and then enhancing the gold label with the IntenSE $M$ kit (Amersham International). Sections were rinsed in $0.1 \mathrm{M}$ glycine $\mathrm{HCl}$ buffer, $\mathrm{pH} 2.5$, for two minutes, in Tris buffer, $\mathrm{pH} 7.2$, for five minutes, and stained for D10 as before.

HISTOLOGICAL CLASSIFICATION OF GASTRIC

CANCERS

The tumours were classified according to Lauren $^{1}$ and the WHO International Histological Classification of Tumours, ${ }^{9}$ either as being of a diffuse or intestinal-type, or of an indeterminate category, based on the predominant histological growth pattern of the tumours seen in the sections stained with antibody 7HGM1D7-B. The indeterminate category refers to tumours with about equal proportions of the diffuse and intestinal growth patterns. ${ }^{9}$ Other histological features of the tumours were recorded as solid, tubular, papillary, or mucinous, ${ }^{9}$ and the general degree of differentiation of the tumours was noted.

HISTOCHEMICAL STAINING

Sections were digested with salivary amylase for one hour at $37^{\circ} \mathrm{C}$, washed in distilled water, stained with Alcian blue, $\mathrm{pH} 2.5$, and then by the periodic acid Schiff (PAS) reaction ${ }^{10}$ for carbohydrate (AB-dPAS stain).

\section{Results}

ANTIGEN EXPRESSION IN NORMAL TISSUE

As described elsewhere, the normal tissue distribution of D10 is limited to gastric mucous neck cells, the glands of the cardia and pylorus, Brunner's glands, peribiliary glands, and the periductal glands of the pancreas; staining for D10 was present as cytoplasmic granules in all samples of the tissues tested. ${ }^{6}$ The distribution of D10 in the 32 specimens of stomach studied here was similar to that reported previously. Figure 1A illustrates the spatial relation of mucous neck cells staining for D10 with cells of the proliferative zone of gastric corpus mucosa, as revealed by staining for PCNA.

The normal tissue distribution of antigen 17NM has previously been shown to be limited to the mucous vacuole of the goblet cells of the small and large intestine. It was demonstrated in all 136 samples of normal colon and 32 of small bowel, in the goblet cells of intestinal metaplasia in six stomachs and in 61 of 101 primary colorectal adenocarcinomas. ${ }^{71}$ $17 \mathrm{NM}$ was not present in the normal mucosa 
of any of the 32 stomachs examined in the current study, but was present in the goblet cells of all examples of intestinal metaplasia seen in eight of the 15 stomachs with a diffuse-type cancer and seven of 13 stomachs with an intestinal-type cancer for which "Swiss rolls" of macroscopically normal mucosa were available for study (table 1).

Antibody 7HGM-1D7-B stained the cytoplasm of the epithelial cells of stomach, intestine, breast, bile ducts, and the transitional epithelium of the kidney calyces, as well as

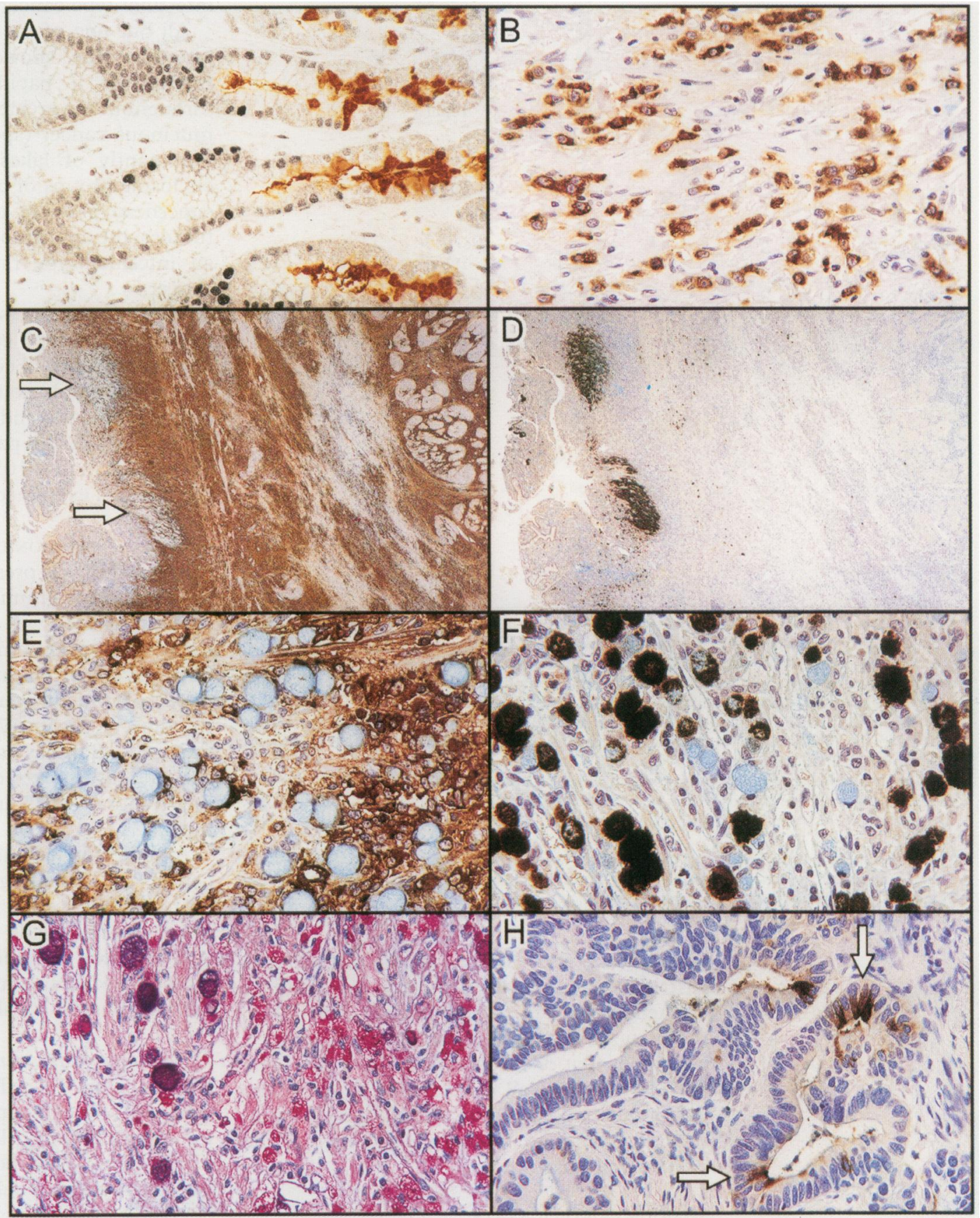

Figure 1 Immunohistochemical staining of sections of human stomach for antigens D10,17NM, and PCNA. (A) Corpus mucosa showing cell nuclei stained for PCNA and mucous neck cells for antigen D10. PCNA positive nuclei (black) are mainly present in the lower pit and isthmus regions and above the mucous neck cells (staining brown for D10). (B) $A$ typical diffuse-type gastric carcinoma (specimen 6 in table 1) showing most tumour cells staining strongly for D10. (C-G) Serial sections of a D10 positive, diffuse-type gastric carcinoma (specimen 8). (C) Shows that most of the cancer cells stain for D10 and are widely disseminated throughout the stomach wall. Two areas (arrows) near the surface of the tumour stain for acid mucins with the Alcian blue counter stain and contain relatively few cells staining for D10. (D) Shows that cells in the two Alcian blue positive areas marked in $C$, together with scattered, solitary cells in the adjacent tissue, stain strongly for antigen 17NM (therefore, this D10 positive tumour includes foci of diffuse tumour cells of the intestinal goblet cell phenotype). (E) $A$ higher power view of $C$ to show part of the deeper edge of one of the Alcian blue stained areas and the presence of numerous large, round, Alcian blue positive cancer cells which, unlike the surrounding smaller tumour cells, do not stain for antigen D10. (F) $A$ higher power view of $D$ corresponding to part of the section area seen in $E$ to show that the majority of the Alcian blue positive, D10 negative cells seen in E stain strongly for 17NM. (G) An area of a section stained with the AB-dPAS stain corresponding to part of that seen in E; this shows the large Alcian blue positive cells seen in $E$ staining purple for acid mucins, and the smaller, adjacent cancer cells in the right half of the photomicrograph (corresponding to the D10 positive cells seen in E) staining red for neutral mucins. (H) An intestinal-type cancer (specimen 13) showing the focal cell staining for D10 (arrows) typical of intestinal-type cancers that express D10. (Original magnifications: $A, B, E, F, G, H \times 250 ; C, D \times 12$.) 
Table 1 Antigen expression and mucin synthesis by 15 diffuse, 16 intestinal, and one indeterminate type gastric carcinomas

\begin{tabular}{|c|c|c|c|c|c|}
\hline \multirow{2}{*}{$\begin{array}{l}\text { Specimen } \\
\text { numbers* }\end{array}$} & \multicolumn{2}{|c|}{ Antigen } & \multirow[b]{2}{*}{ Mucin type } & \multirow{2}{*}{$\begin{array}{l}\text { Other } \\
\text { histological } \\
\text { featurest }\end{array}$} & \multirow{2}{*}{$\begin{array}{l}\text { Intestinal } \\
\text { metaplasia }\end{array}$} \\
\hline & $D 10$ & $17 N M$ & & & \\
\hline \multicolumn{6}{|c|}{ Diffuse type } \\
\hline 6 & $+++\emptyset$ & - & $\mathrm{M}, \mathrm{A}$ & & + \\
\hline 9 & +++ & - & $\mathrm{N}$ & & + \\
\hline 10 & +++ & - & $\mathrm{N}$ & Tubular & + \\
\hline 14 & +++ & - & $\mathbf{N}$ & & - \\
\hline 18 & +++ & - & $\mathrm{N}$ & & - \\
\hline 23 & +++ & - & $\mathbf{M}$ & & - \\
\hline 7 & ++ & - & $\mathrm{N}$ & & - \\
\hline 32 & ++ & - & $\mathbf{M}$ & & + \\
\hline 12 & +++ & + & $M$ & Tubular & + \\
\hline 8 & +++ & ++ & $\mathrm{N}, \mathrm{A}$ & & - \\
\hline 17 & +++ & ++ & $\mathrm{M}, \mathrm{A}$ & & + \\
\hline 2 & + & +++ & A & & + \\
\hline 5 & + & +++ & A & Tubular & - \\
\hline 16 & - & +++ & $\mathbf{M}$ & & + \\
\hline 24 & - & +++ & A & & - \\
\hline \multicolumn{6}{|c|}{ Intestinal type } \\
\hline 25 & ++ & - & $\mathrm{N}, \mathrm{A}$ & Tubular & + \\
\hline 31 & ++ & - & $\mathrm{N}$ & Tubular, pd & - \\
\hline 13 & + & - & A & Papillary & - \\
\hline 11 & + & + & $\mathrm{N}, \mathrm{A}$ & Tubular & + \\
\hline 28 & + & ++ & $\mathrm{N}, \mathrm{M}$ & Tubular, pd & - \\
\hline 3 & - & +++ & $\mathrm{M}$ & Mucinous & - \\
\hline 1 & - & ++ & $M$ & Tubular & NA \\
\hline 15 & - & ++ & $\mathrm{M}, \mathrm{A}$ & Tubular & - \\
\hline 20 & - & ++ & $\mathrm{M}, \mathrm{A}$ & Tubular, pd & NA \\
\hline 21 & - & ++ & $\mathbf{M}$ & Tubular & + \\
\hline 27 & - & ++ & $\mathrm{M}, \mathrm{A}$ & Tubular & + \\
\hline 19 & - & - & - & Papillary & + \\
\hline 22 & - & - & $\mathbf{N}$ & Papillary & + \\
\hline 26 & - & - & - & Tubular, solid & NA \\
\hline 29 & - & - & $\mathrm{N}$ & Tubular, solid & + \\
\hline 30 & - & - & $\mathbf{M}$ & Tubular, solid & - \\
\hline \multicolumn{6}{|c|}{ Indeterminate type } \\
\hline 4 & +++ & + & $\mathbf{M}, \mathbf{A}$ & Solid, diffuse & - \\
\hline
\end{tabular}

$\star$ The specimens are not in order of their accession, but for both diffuse and intestinal types have been arranged in order of their expression of antigen, first for D10 and then for 17NM

†Records the presence of tubules in some areas of the otherwise diffuse-type cancers, and the general histological pattern(s) of tumour growth seen in sections of the intestinal-type cancers. pd, poorly differentiated; solid, growing mainly as solid cords of tumour cells.

IIntestinal metaplasia in the non-neoplastic mucosa as demonstrated by staining of goblet cells with antibody $17 \mathrm{NM}-20-20$. +, metaplasia present; -, metaplasia not found; NA, insufficient mucosa available for evaluation.

$\$$ A subjective estimate of the proportion of cancer cells staining for antigen made by comparison with the numbers of cancer cells seen in an adjacent section stained with antibody 7HGM-1D7-B. - , no cells stained; + , an occasional cell stained; ++ , numerous cells stained; +++ , most cells stained.

qThe predominant histochemical staining of intra- and extracellular mucins by the AB-dPAS stain, seen generally or in different areas of the cancers. -, no staining; A, acid mucin; N, neutral mucin; $M$, a mixture of acid and neutral mucins.

NA, "Swiss rolls" of mucosa not available.

hepatocytes, and the neuroendocrine cells in gastric mucosa. It did not stain the stratified epithelium of skin or oesophagus. Therefore, the tissue distribution of the antigen identified resembles that of cytokeratins 8 and $18 .^{12}$ The antibody stained all cancer cells in all of the gastric adenocarcinomas tested, and was particularly useful in evaluating sections of the diffuse-type carcinomas.

\section{TUMOUR CLASSIFICATION}

Fifteen of the cancers were classified as diffusetype (table 1). These were from 10 men (average age 54 years, median 52) and five women (average age 54 years, median 55), with cancer sites in cardia (one), corpus (four), and antrum (10). Of the remaining 17 tumours, 16 were of the intestinal type and one was indeterminate; they were from 12 men (average age 61 years, median 60) and five women (average age 73 years, median 74), with cancer sites in the cardia (six), corpus (three), and antrum (seven), and one unspecified site.

All 15 tumours of diffuse type consisted predominantly of small, pleomorphic cancer cells widely disseminated throughout the tissue as single cells, or in loose, irregular groups. Three of the diffuse cancers (specimens 5, 10, and 12 in table 1) showed occasional tubules in some areas, which in each case constituted less than $\sim 10 \%$ of the sectioned part of the tumour. These tubules lacked the more ordered columnar cell arrangement of those seen in the intestinal-type cancers.

All but one of the 16 tumours classified as intestinal-type showed well formed glands/ tubules or a papillary pattern of growth throughout the greater part of the tumour. The mucinous cancer (specimen 3) consisted predominantly of lakes of extracellular mucus enclosing groups of cells, but with some more superficial parts of the tumour consisting of tubules, the appearance suggesting an origin in an intestinal-type rather than a diffuse-type cancer. ${ }^{\prime}$

One tumour (specimen 4) was an indeterminate-type carcinoma consisting predominantly of solid cords of cells with one area of papillary and tubular carcinoma, as well as one intramucosal and another submucosal area of a diffuse growth pattern that together represented less than $\sim 10 \%$ of the tumour tissue in the sections examined.

\section{ANTIGEN EXPRESSION IN CANCERS}

Antigen D10 was present in gastric cancer cells as cytoplasmic granules. Granular and diffuse staining for D10 was also seen in the stroma of many areas containing large numbers of D10 positive cancer cells in diffuse-type cancers, but pools of mucin were not present in these tumours. Antigen $17 \mathrm{NM}$ was present in the mucigen granules or mucous vacuoles of cancer cells, in extracellular mucus surrounding tumour cells, and in pools of secreted mucus.

A subjective estimate of the proportions of cancer cells staining for D10 or $17 \mathrm{NM}$ in representative areas of sections of the 32 tumours was made using adjacent sections stained with antibody 7HGM-1D7-B as a measure of total numbers of cancer cells present. Results are shown in table 1.

\section{Diffuse-type gastric cancers}

Eleven of the 15 diffuse-type cancers showed numerous to most cancer cells staining strongly for antigen D10 (fig 1B). When not completely obscured by D10 positive cytoplasmic granules, these tumour cells generally showed some degree of cytoplasmic staining with the Alcian blue counter stain and slight to strong staining for predominantly neutral mucins with the AB-dPAS stain. Sections from three of these 11 diffuse-type tumours (specimens 12, 8, and 17) also showed some areas with occasional to numerous, large signet ring cells with granular cytoplasm that did not stain for D10 but the majority of which stained strongly for antigen 17NM. These large signet ring cells stained predominantly for acid mucins with the AB-dPAS stain, in contrast with the neutral mucins of the D10 positive tumour cells seen throughout the bulk of these three cancers (figs 1C-G). Foci of cancer cells expressing the 
intestinal goblet cell phenotype were, therefore, present in these three otherwise uniformly D10 positive, advanced diffuse-type cancers.

The remaining four diffuse-type gastric cancers were signet ring cell carcinomas from one male and three female patients (specimens 2, 5, 16 , and 24). Sections of these four tumours were characterised by discrete areas showing intense staining for antigen 17NM (fig 2A); these areas corresponded to pools of extracellular mucin staining predominantly for acid mucins with the AB-dPAS stain. 17NM in these areas was also mostly extracellular. The stroma lying outside of the pools of mucin contained few tumour cells in two of these tumours; one contained numerous cancer cells (including signet ring cells) many of which did not stain for 17NM (as shown in figs $2 \mathrm{~A}$ and $\mathrm{B}$ for specimen 2); and the fourth tumour showed innumerable, small, irregularly shaped and diffusely scattered cells all of which appeared to stain for $17 \mathrm{NM}$ in their cytoplasm. Tumour cells in these areas also stained predominantly for acid mucins with the AB-dPAS stain (figs $2 \mathrm{~A}-\mathrm{F}$ ). Two of the four tumours (specimens 2 and 5) showed occasional small groups of cells ( $<40$ cells) staining for D10 in areas outside the pools of mucin and in which tumour cells did not stain for $17 \mathrm{NM}$. Therefore, foci of cancer cells expressing the D10 phenotype were present in these two tumours which otherwise uniformly expressed the intestinal goblet cell phenotype.

\section{Intestinal-type gastric cancers}

Staining for antigens D10 and 17NM was not as consistent in the tumour cells of the intestinal-type cancers as in the diffuse-type and, when present, was generally only focal and seen in occasional, scattered cells (figs $1 \mathrm{H}, 2 \mathrm{G}$ and $\mathrm{H}$ ). Of the 16 intestinal-type cancers, three stained exclusively for D10, two stained for both D10 and 17NM, and another six stained exclusively for $17 \mathrm{NM}$. D10 positive cells stained predominantly for neutral mucins, and 17NM positive cells for acid mucins. The remaining five cancers did not stain for either D10 or $17 \mathrm{NM}$, and showed little or no mucin with the AB-dPAS stain. When present in the same tumour, the two antigens were generally segregated in different regions of the tumours.

Indeterminate-type gastric cancers

In specimen 4, the solid cords of cancer cells and the glandular areas showed the great majority of cells staining for D10, but there were also rare, scattered cells staining for 17NM. The small areas of diffuse-type cancer present in this specimen (see above) did not stain for either $17 \mathrm{NM}$ or D10 antigen, but stained for acid mucins with the AB-dPAS stain.

Colonic signet ring cell carcinomas and adenocarcinomas

None of the four signet ring cell cancers of the colon or the five adenocarcinomas of the colon stained for D10, but all of these tumours except for two of the adenocarcinomas stained for $17 \mathrm{NM}$; the two adenocarcinomas that did not stain for $17 \mathrm{NM}$ did not stain for mucins with the AB-dPAS stain.

\section{Discussion}

Antigen D10 is characteristic of certain cells of organs derived from the foregut that typically synthesise neutral mucins. ${ }^{6}$ It is invariably expressed in normal gastric mucosa and, therefore, could be expected to occur in some mucus secreting gastric tumours. On the other hand, antigen $17 \mathrm{NM}$ is specific to intestinal goblet cells and only appears in the stomach in intestinal metaplasia. Therefore, these two antigens represent universally expressed, mutually exclusive and distinctive tissue specific cell markers of potential use in tracing the cell lineages of gastric and intestinal adenocarcinomas. In the following discussion, we will refer to the D10 phenotype as that of a mucous neck cell, although the similarity in cytology of mucous neck cells to the mucous cells of the cardiac, pyloric and Brunner's glands is well documented $^{1314}$ and further supported by the presence of D10 in each of these tissues. ${ }^{6}$ Cells staining for $17 \mathrm{NM}$ will be referred to as of an intestinal goblet cell phenotype.

This study has shown that the majority $(84 \%)$ of gastric carcinomas display one or both of the cell phenotypes investigated, and that there is some correlation between the predominant phenotype displayed and the histological classification of the tumours. As both D10 and $17 \mathrm{NM}$ are normally associated with mucin producing cells, the generally lower prevalence of antigen positive cells in the intestinal-type cancers was probably related to their generally poorer mucin content as demonstrated by histochemical staining for mucins. The four diffuse cancers that showed extensive pools of extracellular mucin, as well as the one intestinal-type mucinous cancer, all displayed predominantly the intestinal goblet cell phenotype, and this was in keeping with the active secretion of acid mucins normally exhibited by this cell phenotype. In contrast, the diffuse cancers with cells exclusively or predominantly expressing D10 did not show pools of extracellular mucin except in those cancers containing segregated cells of the intestinal goblet cell phenotype (as illustrated in fig 1D). The two phenotypes studied revealed that Lauren's diffuse-type cancers are a heterogeneous group that can be subdivided into cancers that exclusively express either the mucous neck cell or intestinal goblet cell (signet ring cell) phenotype, or both phenotypes in segregated areas. As either antigen can be expressed in a diffuse or intestinal-type cancer, the phenotypes do not strictly characterise histological tumour type. However, if the apparent differences in prevalence of the two phenotypes in diffuse and intestinal-type cancers are real, the differences in behaviour of two such disparate cancer cell phenotypes could help to explain the generally accepted biological relevance of Lauren's tumour classification.

All of the diffuse-type gastric carcinomas displayed predominantly one or other of the two cell phenotypes. Generally, the two antigen markers and associated mucin profiles dis- 
played by the malignant cells matched those of the two normal cell types (the mucous neck cell and the intestinal goblet cell), suggesting that many tumour cells in the diffuse-type cancers were fully differentiated, at least with respect to these tissue specific components. Because of their continued synthesis of mucins, Lauren suggested that the cells of diffuse-type cancers are rather more biochemically mature than their lack of cell cohesion would indicate, ${ }^{1}$ and the continued expression of D10 by the majority of these cancers supports such an interpretation. However, there were many cancer cells that did not stain for D10 or 17NM in sections of most of the diffuse-type cancers, and it is these unstained cells that may be the

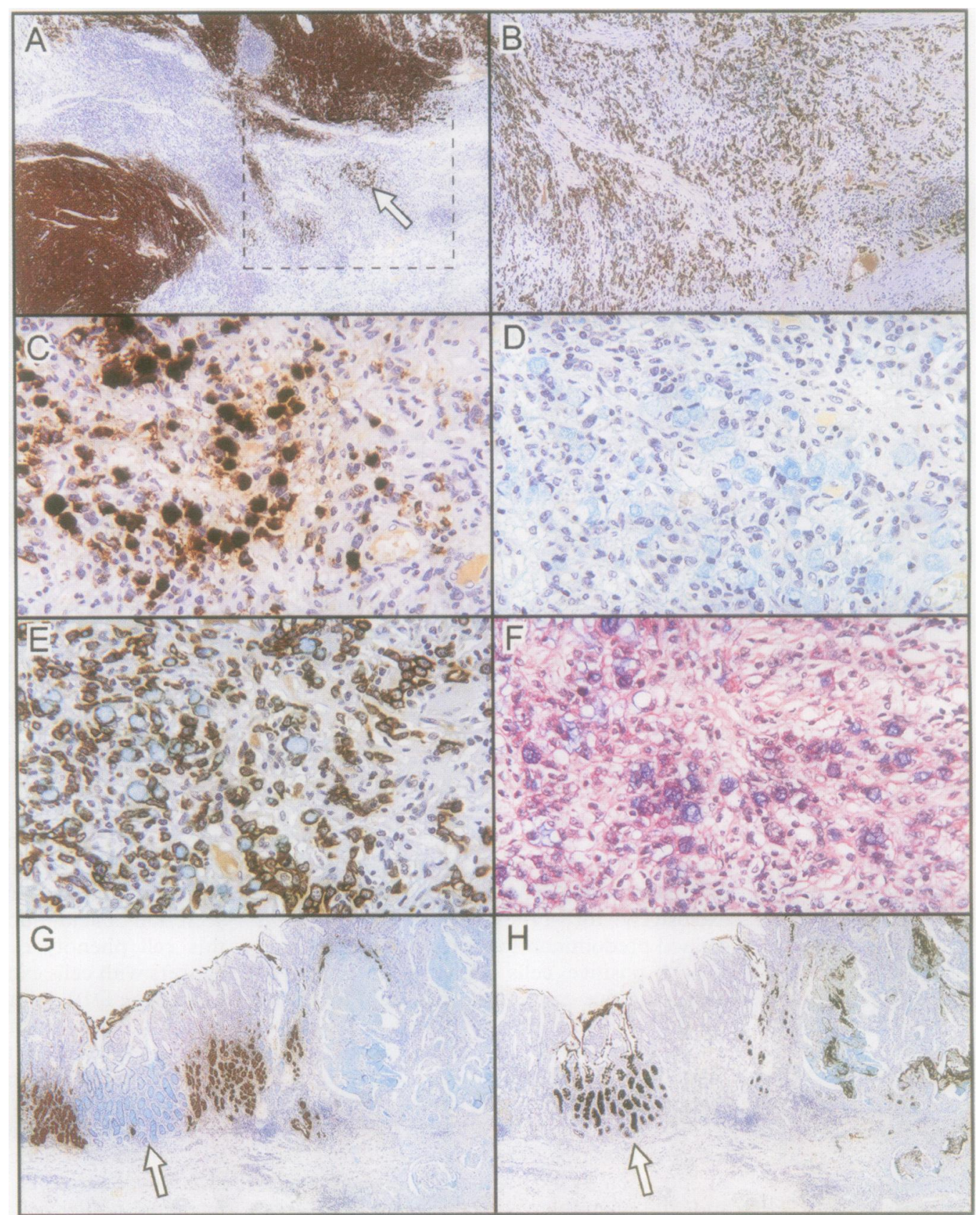

Figure 2 Immunohistochemical staining of sections of human stomach for antigens D10 and 17NM. $(A-F)$ Serial sections of a 17NM positive diffuse-type gastric carcinoma (specimen 2 in table 1). (A) Low power view showing areas containing cancer cells and pools of extracellular mucin (lower left and upper right) staining strongly for antigen 17NM. In an adjacent section (not shown), stained with $A B-d P A S$, these 17NM positive areas stained strongly for acid mucins. Scattered groups of cancer cells showing cytoplasmic staining for 17NM (arrow) are also present in the generally unstained stroma between the pools of mucin. (B) The area corresponding to that outlined in $A$ in a section stained with antibody 7HGM-1D7-B to show the presence of numerous cancer cells in the stroma, many of which did not stain for 17NM in section A. (C) $A$ higher power view of the area marked by the arrow in section $A$ to show a group of the 17NM positive cancer cells. (D) The area corresponding to that in $C$ in a section stained for antigen D10 to show large signet ring cells similar in size to the 17NM positive cells in C staining for acid mucins with the Alcian blue counterstain but not for D10. $(E)$ The area corresponding to that in $C$ and $D$ in a section stained with the $7 H G M-1 D 7-B$ antibody showing that there are many other smaller cancer cells present besides the large, Alcian blue positive signet ring cells. $(F)$ The area corresponding to that in $C, D$, and $E$ in a section stained with $A B-d P A S$ showing that the large signet ring cells contain predominantly acid mucins. ( $G$ and $H$ ) Serial sections of an intestinal-type gastric cancer (located in the right half of the mucosa and submucosa) and adjacent mucosa that contains normal pyloric glands and an area of intestinal metaplasia (arrow). (G) Shows the pyloric glands staining for D10 while the area of intestinal metaplasia (arrow) and the carcinoma are unstained. $(H)$ Shows the reverse, with the normal pyloric glands unstained for 17NM, but goblet cells in the intestinal metaplasia staining for $17 N M$ and focal staining of the carcinoma. (Original magnifications: $A \times 25 ; B \times 60 ; C, D, E, F$ $\times 250 ; G, H \times 15$.) 
most active in proliferation. In the case of the four diffuse-type cancers with tumour cells predominantly or exclusively of a phenotype foreign to the stomach, one could argue that the cells have arisen from the same cell lineage as that giving rise to intestinal metaplasia in the stomach. Intestinal metaplasia has been reported to be more frequent and widespread in stomachs with intestinal-type cancers than in those with the diffuse-type, ${ }^{14}$ although the relation remains uncertain. ${ }^{15} \mathrm{We}$ found intestinal metaplasia in almost $50 \%$ of the stomachs bearing each type of cancer as demonstrated by staining for the 17NM antigen in "Swiss rolls" of gastric mucosa from this small series of 32 stomachs. These proportions need to be confirmed in a larger series of cancers.

The consistent expression of D10 by the majority of the diffuse-type cancers suggests that this group of tumours could have arisen from the cell lineage that normally differentiates into mucous neck cells and the glands of cardia and pylorus. Previous investigators have identified dysplasia in the proliferative zone of non-metaplastic gastric glands and proposed this as the site of origin of diffuse-type cancers. ${ }^{16}$ Also, histological and histochemical evidence that the mucous neck cell may give rise to diffuse-type carcinoma has been presented by others, ${ }^{17}$ and similarity in lectin staining and in histochemical and ultrastructural features between normal mucous neck cells and the neoplastic cells demonstrated. ${ }^{18}$ Our previous observations on cell proliferation in normal human gastric mucosa suggest that mucous neck cells are mature cells that rarely divide, although pre-mucous neck cells in the isthmus that show some cytoplasmic granules staining for D10 can do so. ${ }^{19}$

It should be noted that D10 is also characteristic of pyloric gland metaplasia found in inflammatory conditions of the stomach, small bowel, biliary tree, and gall bladder. ${ }^{6}$ This form of metaplasia is said to be derived from an ulcer associated cell lineage (UACL) distributed throughout the gastrointestinal tract, which, in response to mucosal ulceration, buds from the base of glands and then proliferates to form the metaplastic glands. ${ }^{20}$ Antigen D10 has been demonstrated in the UACL in the small intestine. ${ }^{6}$ The UACL has been described as having the differentiation programme of Brunner's glands but acquiring the proliferative organisation of the gastric gland. ${ }^{21}$ If the UACL is distinct from the gastric stem cell lineage normally giving rise to mucous neck cells, it is possible that D10 positive, diffuse-type cancers may also arise indirectly, that is by transformation of cells of the UACL as they proliferate in response to the chronic gastritis which frequently precedes gastric carcinoma. The UACL does not stain for $17 \mathrm{NM}$ and is not known to be directly associated with intestinal metaplasia or intestinal goblet cells, and is unlikely therefore to give rise to gastric cancers other than those displaying antigen D10.

The small group of four diffuse-type cancers that were distinguished by predominantly or exclusively displaying the $17 \mathrm{NM}$ intestinal goblet cell phenotype and secreting acid mucins histologically were classic signet ring cell carcinomas. ${ }^{9}$ The cell phenotype displayed by these four tumours suggests a relation with, and perhaps origin in, intestinal metaplasia, this being the only non-neoplastic tissue in the stomach to express the 17NM antigen. Intestinal metaplasia is reported to arise from within the isthmus of the glands of intact gastric mucosa, ${ }^{22}$ or during the reparative process of erosions. ${ }^{23}$ In the early stages of the development of intestinal metaplasia in intact mucosa, the proliferative zone may remain in the isthmus with the surface epithelium and pits showing metaplastic cells in continuity with the deeper normal gastric glands. ${ }^{22}$ Transformed cells developing at such a site could give rise to $17 \mathrm{NM}$ positive diffuse-type carcinomas in an analogous fashion to the D10 positive diffuse cancers that may develop from pre-mucous neck cells, as suggested above. Since there was evidence of tubule formation in three of the 15 diffuse-type carcinomas, it is also possible that some of the tumours studied were originally intestinal-type cancers that were then overgrown by a diffuse-type tumour, although there was no histological evidence to support this.

Both the D10 and 17NM cell phenotypes were co-expressed in individual tumours of a quarter of all the gastric cancers examined. Since no more than $10 \%$ of subjects with gastric carcinoma are reported to show macroscopically discrete synchronous cancers, ${ }^{24}$ collision tumours are an unlikely explanation of the mixed cell phenotypes found in some of our specimens. A stratification of tumour cells which parodies normal gastric cell types has been observed in the early stages of development of gastric carcinoma by other investigators, ${ }^{25}$ and segregation of the two cell phenotypes was noted in the mucosa of some of the diffuse-type cancers studied here. Therefore, it appears that some transformed gastric cells are sufficiently plastic and primitive to be capable of divergent differentiation into either the D10 or $17 \mathrm{NM}$ phenotype. Clonal selection could account for the predominance with time of one or other phenotype or, when there is no selective advantage, the growth of both cell phenotypes would result in the regional differences in phenotype expression that we observed within some individual tumours.

Since normal Brunner's glands form by budding off from the crypts of the duodenal mucosa, ${ }^{26}$ the stem cells in the crypts of the duodenum must be capable of divergent differentiation into either mucosa containing intestinal goblet cells (displaying antigen $17 \mathrm{NM}$ ) or Brunner's glands (displaying antigen D10). It appears that in the course of development of diffuse-type cancers the transformed gastric stem cells consistently regain such plasticity and go on to express one or both of the two phenotypes studied. Why the mucous neck cell phenotype rather than that of the intestinal goblet cell is then more frequently expressed by such cancers may be explained on the basis that this would be the more normal direction in differentiation for gastric cells (differentiation into mucous neck cells or mucous cells of the 
cardiac and pyloric glands). The co-existence of both phenotypes found in some individual cancers is also in keeping with a capacity for divergent differentiation by the neoplastic cells, and helps to explain the confusing histochemical heterogeneity of these tumours.

This study has shown that gastric adenocarcinomas are characterised by two cell phenotypes, one of which is foreign to the normal stomach mucosa and both of which are normally present in the duodenum. Reversion of the transformed gastric stem cell to a more primitive gut stem cell lineage possessing the same capacity for divergent differentiation as the normal duodenal stem cell is the most economical explanation of the consistent association of the two phenotypes with the noncohesive diffuse-type carcinoma. These cancers typically show one of the two antigens predominating, usually D10, and being strongly expressed by the majority of the cancer cells. In contrast, intestinal-type cancers with their more ordered tubular structure more frequently display the intestinal goblet cell phenotype and typically express the antigens in relatively few tumour cells.

The work was supported by a grant from the National Health and Medical Research Council of Australia. This work was presented in part at the Australian Gastroenterology Week 1996, sented in part at the Australian Gastroenterology Week 1996,
the Annual Scientific Meeting of the Gastroenterological Socithe Annual Scientific Meeting of the Gastroenterological Soci-
ety of Australia, held in Adelaide, September 1996, and ety of Australia, held in Adelaide, September 1996, and
published in abstract form in $\mathcal{F}$ Gastroenterol Hepatol 1996;11 (Suppl):A94

1 Lauren $P$. The two histological main types of gastric carcinoma: diffuse and so-called intestinal-type carcinoma Acta Path Microbiol Scand 1965;64:31-49.

2 Correa P. The epidemiology of gastric cancer. World f Surg 1991;15:228-34.

3 Janssen CW Jr, Lie RT, Maartmann-Moe H, Matre R. The influence of age on the growth and spread of gastric carcinoma. Br f Cancer 1991;63:623-5.

4 Ming SC, Goldman H, Freiman DG. Intestinal metaplasia and histogenesis of carcinoma in human stomach. Cancer and histogenesis

5 Stefanini M, De Martino C, Zamboni L. Fixation of ejaculated spermatozoa for electron microscopy. Nature 1967; 216:173-4

6 Hughes NR, Bhathal PS, Francis DMA. Phenotypic identity of gastric mucous neck cells and mucous cells of cardiac, pyloric, and Brunner's glands. F Clin Pathol 1994;47:53-7.

7 Hughes NR, Walls RS, Newland RC, Payne JE. Antigen expression in normal and neoplastic colonic mucosa: three tissue-specific antigens using monoclonal antibodies to isolated colonic glands. Cancer Res 1986;46:2164-71.
8 Cattoretti G, Pileri S, Parravicini C, Becker MHG, Poggi S, Bifulco $\mathrm{C}$, et al. Antigen unmasking on formalin-fixed, paraffin-embedded tissue sections. $\mathcal{F}$ Pathol 1993;171:8398.

9 Watanabe $\mathrm{H}$, Jass JR, Sobin $\mathbf{L H}$, in collaboration with pathologists in eight countries. Histological typing of oesophageal and gastric tumours. In: WHO international histological classification of tumours. Berlin: Springer-Verlag, histological clas.

10 Cook HC. Carbohydrates. In: Bancroft JD, Stevens A, eds. The theory and practice of histological techniques. Edinburgh: Churchill Livingstone, 1982:180-216.

11 Hughes NR. Gland heterogeneity and colorectal neoplasia. University of Sydney: $\mathrm{PhD}$ thesis, 1988.

12 Moll R, Franke WW, Schiller DL, Geiger B, Krepler R. The catalog of human cytokeratins: patterns of expression in normal epithelia, tumors and cultured cells. Cell 1982;31 11-24

13 Bloom W, Fawcett DW. A textbook of histology. Philadelphia: WB Saunders Company, 1975:639-57.

14 Katsuyama T, Spicer SS. Histochemical differentiation of complex carbohydrates with variants of the concanavalin A-horseradish peroxidase method. $\mathcal{f}$ Histochem Cytochem 1978;26:233-50.

15 Dixon MF. Progress in gastric cancer. In: Kirkham N, Hall PA, eds. Progress in pathology. Vol 1. Edinburgh: Churchill Livingstone, 1995:13-29.

16 Ghandur-Mnaymneh L, Paz J, Roldan E, Cassady J. Dysplasia of nonmetaplastic gastric mucosa. A proposal for its classification and its possible relationship to diffuse-type gastric carcinoma. Am $\mathcal{F}$ Surg Pathol 1988;12:96-114.

17 Yamashina M. A variant of early gastric carcinoma. Histologic and histochemical studies of early signet ring Histologic and histochemical studies of early signet ring
cell carcinomas discovered beneath preserved surface

18 Tatematsu M, Furihata C, Katsuyama T, Miki K, Honda H, Konishi Y, et al. Gastric and intestinal phenotypic expressions of human signet ring cell carcinomas revealed by their biochemistry, mucin histochemistry, and ultrastructure. Cancer Res 1986;46: 4866-72.

19 Hughes NR, Bhathal PS. Do gastric mucous neck cells divide? [abstract]. Int $\mathcal{f}$ Surg Pathol 1996;3:217.

20 Wright NA, Pike C, Elia G. Induction of a novel epidermal growth factor-secreting cell lineage by mucosal ulceration in human gastrointestinal stem cells. Nature 1990;343:825.

21 Ahnen D, Poulsom R, Stamp GWH, Elia G, Pike C, Jeffery $\mathrm{R}$, et al. The ulceration-associated cell lineage (UACL) reiterates the Brunner's gland differentiation programme but acquires the proliferative organization of the gastric gland. $\mathcal{F}$ Pathol 1994;173:317-26.

22 Hattori T, Fujita S. Tritiated thymidine autoradiographic study on histogenesis and spreading of intestinal metaplasia in human stomach. Path Res Pract 1979;164:224-37.

23 Mukawa K, Nakamura T, Nakano G, Nagamachi Y. Histopathogenesis of intestinal metaplasia: minute lesions of intestinal metaplasia in ulcerated stomachs. $\mathcal{F}$ Clin Pathol 1987;40:13-8.

24 Kosaka T, Miwa K, Yonemura Y, Urade M, Ishida T, Takegawa $\mathrm{S}$, et al. A clinicopathologic study on multiple gastric cancers with special reference to distal gastrectomy. Cancer 1990;65:2602-5.

25 Fujimori Y, Akamatsu T, Ota H, Katsuyama T. Proliferative markers in gastric carcinoma and organoid differentiation. Hum Pathol 1995;26:725-34.

26 Krause WJ, Leeson CR. The origin, development and differentiation of Brunner's glands in the rat. $\mathcal{F}$ Anat 1967; 101:309-20. 